\title{
On the oversized problem of Dickey-Fuller-type tests with GARCH errors
}

\author{
Jen-Je Su \\ Griffith University \\ Australia
}

\begin{abstract}
$\underline{\text { Abstract }}$
This paper examines the finite-sample size of a class of Dickey-Fuller-type tests in the presence of GARCH errors, with and without the influence of initial conditions of the underlying simulated path. Oversizing is observed for all tests when the GARCH process is nearly degenerate and the volatility parameter is large, but the degree of size distortion varies across tests and is contingent on the initial condition. The result due to the initial effect is linked to the size distortion caused by a sequence of small downward variance breaks arising in the early stage of the underlying process.
\end{abstract}

Keywords: GARCH; Unit root tests; Size distortion; Initial effect; Monte Carlo experiment

JEL Codes: C22

Running Head: Testing for a unit root under GARCH

Correspondence to:

Jen-Je Su

Department of Accounting, Finance and Economics

Nathan Campus, Griffith University

170 Kesssels Road, Nathan

Brisbane, Queensland 4111, Australia

Email: j.su@griffith.edu.au

Phone: 61-7-37353837

Fax: 61-7-37357760 


\section{[1] Introduction}

It is a well known fact that the Dickey-Fuller (DF) test tends to over-reject the null of a unit root in the presence of GARCH errors. Kim and Schmidt (1993) first investigated the reliability of the DF test in this regard. They concluded that the DF test is generally oversized and that the problem becomes serious when the underlying GARCH process is nearly degenerate, nearly integrated and the volatility parameter is relatively large - that is, when the GARCH effect is “strong”. Similar results were found in Haldrup (1994) and Lin et al. (2003). Valkanov (2005) also demonstrated that with strong GARCH effects, while the small-sample DF distribution appears to converge to the asymptotic distribution, the convergence is rather slow. Because macroeconomic and financial time series are typically characterized by strong GARCH effects, it may rise suspicion about the result in the standard practice of the DF test in empirical work.

The over-rejection problem appears in a class of modified DF tests too. According to Cook (2006), where the standard DF test and several modified DF tests were examined, while on the whole the modified tests appear to be robust to GARCH, the advantage of using them over the standard test is moderate. When the sample size gets larger, since the standard DF test statistic moves closer to its asymptotic distribution, the advantage of the modified tests becomes even less noticeable. Similar to Kim and Schmidt (2003), Cook (2006) identified that the over-sizing of all DF-type tests is mainly driven by the volatility rather than the persistence of the variance process. 
An important but commonly neglected or inadequately treated issue concerning the oversizing problem for unit-root tests is the effect of initial values of a simulated I(1)-GARCH series. Kim and Schmidt (1993), Lin et al. (2003), Valkanov (2005) and Cook (2006) did not deal with such an effect in their Monte Carlo exercises. Haldrup (1994) is the only paper in the unit root test literature that dealt with this effect. In order to get rid of the effect Haldrup (1994) suggested generating additional ten observations for each simulating path and then deleted the first ten from the path. Although such a rule seems to work well in the elimination of the initial effect when the GARCH effect is moderate, it does not work well when the effect becomes "strong”. Lee and Tse (1996), in their study regarding cointegration tests, argued that when the GARCH process is nearly integrated and nearly degenerate the simulated process takes a long while to settle down. In such a case discarding the first 500 observations is required to get rid of the initial effect. Though the same simulation rule should be adopted for unit root tests, it has been largely ignored in the unit-root literature.

In this paper, extensive Monte Carlo simulations are conducted to examine the influence of initial values of a simulated I(1)-GARCH process to several DFtype unit root tests. Basically, it is found that the over-rejection problem for the standard DF test with GARCH errors can become much more serious if the initial effect is not trimmed. In contrast, the initial effect does rather limited impact to the modified tests. The result due to the mixed effect of GARCH and the initial conditions is linked to the literature of Kim et al. (2002) and Cook (2003, 2004) 
where the oversizing problem for the DF-type tests is caused by an early break in the innovation variance.

The paper proceeds as follows. In section 2, several DF-type tests are presented. Section 3 states design and reports results of the Monte Carlo experiment. Section 4 concludes.

\section{[2] Dickey-Fuller-type Tests}

Consider the model

$$
y_{t}-\mu=\rho\left(y_{t-1}-\mu\right)+\varepsilon_{t},
$$

where $y_{t}, \mathrm{t}=1,2, \ldots, \mathrm{T}$, are observations, $\varepsilon_{t}$ is a serially-uncorrelated zero-mean process, and $\mu, \rho$ are unknown parameters. Let $\dot{y}_{t}=y_{t}-\bar{y}$ and $\bar{y}=T^{-1} \sum_{t=1}^{T} y_{t}$. The standard DF test tests the unit root hypothesis $H_{0}: \rho=1$ against the alternative $H_{1}:|\rho|<1$ using a test statistic

$$
\tau=(\hat{\rho}-1) / \operatorname{se}(\hat{\rho})
$$

where $\hat{\rho}=\sum_{t=2}^{T} \dot{y}_{t} \dot{y}_{t-1} / \sum_{t=2}^{T} \dot{y}_{t-1}^{2}$ is the OLS estimator of $\rho$ in (1) and $\operatorname{se}(\hat{\rho})$ is its standard error. Despite its popularity, the DF test is known to have low power when $\rho$ is close to one.

Several attempts have been made to improve the power of the standard DF test in the literature. Pantula et al. (1994) introduced a weighted symmetric (WS) estimator

$$
\hat{\rho}_{w s}=\sum_{t=2}^{T} \dot{y}_{t} \dot{y}_{t-1} /\left(\sum_{t=2}^{T-1} \dot{y}_{t}^{2}+T^{-1} \sum_{t=1}^{T} \dot{y}_{t}^{2}\right)
$$


of $\rho$ in (1). Based on the WS estimator, they suggested a modified DF test (DFWS):

$$
\tau_{w S}=\sigma_{w s}^{-1}\left(\hat{\rho}_{w s}-1\right)\left(\sum_{t=2}^{T-1} \dot{y}_{t}^{2}+T^{-1} \sum_{t=1}^{T} \dot{y}_{t}^{2}\right)^{1 / 2}
$$

where $\quad \sigma_{w s}^{2}=(T-2)^{-1}\left[\sum_{t=1}^{T-1} w_{t}\left(\dot{y}_{t}-\hat{\rho}_{w s} \dot{y}_{t-1}\right)^{2}+\sum_{t=2}^{T}\left(1-w_{t+1}\right)\left(\dot{y}_{t}-\hat{\rho}_{w s} \dot{y}_{t+1}\right)^{2}\right] \quad$ with $w_{t}=(t-1) / T$. Basically, the power gain of this test comes from the utilization of the reverse autoregressive (AR) representation in addition to the forward AR representation.

Power improvement may also be achieved using different mean-adjustment schemes other than OLS as in the standard DF test. Elliot et al (1996) proposed a modified test using the application of local-to-unity demeaning via generalized least squares (GLS) estimation. The locally demeaned series $\ddot{y}_{t}$ is derived as $\ddot{y}_{t}=y_{t}-\hat{\beta}$ where $\hat{\beta}$ is obtained by regressing $y_{\alpha}=\left(y_{1}, y_{2}-\alpha y_{1}, \ldots, y_{T}-\alpha y_{T-1}\right)^{\prime}$ on $z_{\alpha}=(1,1-\alpha, \ldots, 1-\alpha)^{\prime}$ with $\alpha=1-(7 / T)$. The resulting unit root test, denoted as DF-GLS, is the t-ratio of $\phi$ from the regression: $\Delta \ddot{y}=\phi \ddot{y}_{t-1}+\varepsilon_{t}$. On the other hand, Shin and So (2001) suggested the use of recursively adjusted mean $\bar{y}_{t}=t^{-1} \sum_{i=1}^{t} y_{i}$. The recursively mean-adjusted version of DF test (DF-REC) has the same format as (2), except that $\hat{\rho}$ and $\operatorname{se}(\hat{\rho})$ are obtained from the regression of $\left(y_{t}-\bar{y}_{t-1}\right)$ on $\left(y_{t-1}-\bar{y}_{t-1}\right)$.

While the power advantage of the above mentioned modified DF tests has been confirmed in subsequent studies and become recognized in applied research 
(see, for example, Leybourne et al. (2005)), their robustness in the presence of GARCH has been discussed only very recently in Cook (2006).

\section{[3] Monte Carlo Experiment and Results}

The data-generating process to be considered is a driftless integrated process with GARCH innovations. The process is

$$
y_{t}=y_{t-1}+\varepsilon_{t}, \quad \mathrm{t}=1, \ldots, \mathrm{T}+\mathrm{d},
$$

where $\varepsilon_{t}$ is assumed to be a GARCH process: $\varepsilon_{t}=h_{t} \eta_{t}$, where $\eta_{t}$ is i.i.d. $\mathrm{N}(0,1)$,

$$
h_{t}=\phi_{0}+\phi_{1} \varepsilon_{t-1}^{2}+\phi_{2} h_{t-1} \text {. }
$$

GARCH parameters - typically, $\phi_{1}$ is know as the volatility parameter and $\phi_{2}$ the persistence parameter - are set to reflect the empirical findings. Specifically, $\phi_{1}$ is set between 0 and 0.349 and $\phi_{2}$ between 0.65 and 0.95 , but $\phi_{1}+\phi_{2}$ never goes larger than 0.999, and $\phi_{0}$ is calibrated to make the unconditional variance equal 1 in all cases: $\phi_{0}=1-\phi_{1}-\phi_{2}$. The initial value of the unit-root process is set $y_{0}=0$

and the initial variance $h_{0}=1$. The pseudo random innovations $\left\{\varepsilon_{t}\right\}_{t=1}^{T+d}$ are drawn from $N\left(0, h_{t}\right)$ and used to construct the unit-root process for $\mathrm{T}=100,500,1000$, 2000 and $d=500$. Two sets of series are used for unit-root testing: series [1] contains observations from 1 to $\mathrm{T}$, and series [2] from $\mathrm{d}+1$ to $\mathrm{T}+\mathrm{d}$. In the first series, the initial effect is left untreated while the second series, according to Lee 
and Tse (1996), the initial effect is off-loaded. ${ }^{1}$ All simulations are based on 10,000 replications and done by GAUSS. All rejection frequencies are calculated at the nominal 5\% significance level. ${ }^{2}$ The Monte Carlo results are reported in Table 1.

We summarize and comment here the results in Table 1. First, the simulation result shows that in some cases the difference of size distortion between the standard DF test and the modified tests can be substantially large. In particular, as the sum of GARCH parameters becomes extremely close to the boundary of integration and the volatility parameter is relatively large (say, $\phi_{1}+\phi_{2} \geq 0.99$ and $\phi_{1} \geq 0.24$ ), while the oversizing problem of the standard DF test deteriorates considerably, the modified tests only get slightly worse. For example, when $\left(\phi_{1}, \phi_{2}\right)=(0.349,0.65)$ and $\mathrm{T}=500$, the rejection frequency of the standard DF test is 0.358 , but $0.087,0.146$ and 0.109 for DF-GLS, DF-WS and DF-REC, respectively. Also, the tests tend to show different patterns of size distortion corresponding to T. DF test is less distorted when T is larger if $\phi_{1}+\phi_{2} \leq 0.995$ and becomes irregular if $\phi_{1}+\phi_{2}=0.999$; the DF-GLS test seems unaffected by T; the DF-WS and the DF-REC tests appear more and then less distorted as T gets larger.

Second, the Monte Carlo result shows that the influence of initial conditions for the data generation process may contribute a significant proportion of the size distortion for the standard DF test. Indeed, in some cases, the over-rejecting

\footnotetext{
${ }^{1}$ The appropriateness of this elimination rule has also been confirmed by the author using simulation with a wide class of GARCH models. The result is available upon request

${ }^{2}$ Critical values are obtained by simulation with 50,000 draws for each test at different sample sizes and available upon request.
} 
problem due to GARCH might look much more serious than it actually is provided that such an influence has been eliminated. For example, the rejection rate drops from 0.358 to 0.108 for $\left(\phi_{1}, \phi_{2}\right)=(0.349,0.65)$ at $\mathrm{T}=500$ when the effect is gone. Accordingly, failing to notice the initial effect, the severe over-rejection of the standard DF test with strong GARCH errors is likely to be overstated. On the contrary, the initial effect does not inflate the size of the DF-GLS and the DF-REC tests and only causes a bit more size distortion for the DF-REC test. Besides, without the initial effect all tests seem very stable over a wide range of sample size, implying that the tests converge to the asymptotic distribution rather fast.

Third, even if the initial effect is removed, the GARCH effect itself still brings about some size distortion for the DF-type tests. Again, since the unconditional kurtosis increases as $\phi_{1}$ increases when $\phi_{1}+\phi_{2}$ is fixed, given the value of $\phi_{1}+\phi_{2}$, the size distortion is usually larger with a larger $\phi_{1}{ }^{3}$ Size improvement of the modified DF tests (with the DF-REC test as an exception) over the standard DF test is obvious, but not very striking. The DF-GLS test appears to be the most robust one. In the presence of GARCH, the DF-GLS test tends to over-reject but the rejection frequency never goes larger than 0.088 and this happens only when the GARCH effect is extremely strong.

Finally, it is worth noting that the simulation result due to the initial effect can actually be related to the oversized problem for the same class of unit-root tests considered previously when there exists a variance break. According to Kim et al. (2002), the standard DF test severely oversizes when applied to a unit-root

\footnotetext{
${ }^{3}$ The unconditional kurtosis for an GARCH(1,1) is $3\left[1-\left(\phi_{1}+\phi_{2}\right)^{2}-2 \phi_{1}^{2}\right]^{-1}\left[1-\left(\phi_{1}+\phi_{2}\right)^{2}\right]$.
} 
process experiencing an abrupt decrease in innovation variance if the decrease is large and occurs in the early stage of the process. In contrast, as shown in Cook (2003, 2004), the modified DF tests seem quite robust to such a break. As for the case of GARCH, Lee and Tse (1996) noted that when a GARCH model is nearly degenerate and with a relative large volatility parameter the initial variance $h_{0}=1$ is too far in the right tail of its stationary distribution, so the simulated timevarying variance $h_{t}$ tends to decline as t gets larger and this declining will last for a while. In other words, if a simulated GARCH series is untrimmed it will behave like a path arisen from a setting where innovation variance undergoes a sequence of downward breaks soon after the start and the breaks become larger and last longer when the GARCH process is closer to degenerate and with a larger volatility parameter. This should help to explain the puzzling size properties regarding the standard DF test and the modified tests in the presence of GARCH, with and without the initial effect.

Of course, Kim et al. (2002) and Cook (2003, 2004) obtain their results only concerning a sudden large drop in variance, but it is not unreasonable to expect that similar results should occur for a series of small but prolonging variance reduction arising in the early stage of the unit-root path. As a matter of fact, according to the simulation result in Table 2 (please see Appendix I for details) early downward can cause significant size distortion for the standard DF test regardless of whether the breaks are one-shot or sequential. In contrast, variance breaks of all sorts result in much less size distortion for the modified DF tests. 


\section{[4] Conclusion}

In this paper, extensive Monte Carlo simulations are carried out to study the size performance of a class of Dickey-Fuller tests in the presence of GARCH errors, with and without the influence of initial values of the underlying process. In addition to the standard DF test, three modified DF tests are considered. Basically, simulation results show that the compound effect from GARCH and initial conditions can cause significant upward size distortion for the standard DF test but the problem is much less severe if the effect is solely from GARCH. In contrast, the modified tests seem insensitive to the initial effect. Even if the initial effect has been suitably controlled, all tests suffer size distortion caused by GARCH to some degree. Among the tests, the DF-GLS test seems to have the least size distortion.

\section{$\underline{\text { References }}$}

Cook, S., 2003, Size and power properties of powerful unit root tests in the presence of variance breaks. Physica A 217, 432-448.

Cook, S., 2004, Finite-sample properties of the GLS-based Dickey-Fuller test in the presence of breaks in innovation variance. Austrian Journal of Statistics 33, 305-314.

Cook, S., 2006, The robustness of modified unit root tests in the presence of GARCH, Quantitative Finance 6, 359-363.

Dickey, D., Fuller, W., 1979. Distribution of the estimators for autoregressive time series with a unit root. Journal of the American Statistical Association 74, 427431.

Elliott G., Rothenberg, T.J., Stock J.H. 1996. Efficient tests for an autoregressive unit root. Econometrics 64, 813-836. 
Haldrup, N., 1994, Heteroskedasticity in non-stationary time series: some Monte Carlo evidence. Statistical Papers 35, 297-307.

Kim K., Schmidt, P., 1993. Unit root tests with conditional heteroskedasticity. Journal of Econometrics 59, 287-300.

Kim T., Leybourne S., Newbold, 2002, Unit root tests with a break in innovation variance, Journal of Econometrics 109, 365-387.

Lee, T.H., Tse, Y., 1996, Cointegration tests with conditional heteroskedasticity. Journal of Econometrics 73, 401-440.

Leybourne, S., Kim, T. and Newbold, P., 2005. Examination of some more powerful modifications of the Dickey-Fuller test. Journal of Time Series Analysis 26, 355-369.

Ling, S., Li, W.K., McAleer, M., 2003. Estimation and testing for unit root process with GARCH(1,1) errors: theory and Monte Carlo evidence. Econometric Reviews 22, 179-202.

Pantula, S. Gonzalez-Frarias, G., Fuller, W., 1994. A comparison of unit root test criteria. Journal of Business and Economic Statistics 8, 153-162.

Shin, D., So, B., 2001. Recursive mean adjustment for unit root tests. Journal of Time Series Analysis 5, 595-612.

Valkanov, R., 2005. Functional central limit theorem approximations and the distribution of the Dickey-Fuller test with strongly heteroskedastic data. Economics Letters 86, 427-433. 


\section{Appendix I}

To study the empirical size of the DF-type tests with breaks in innovation variance, a unit-root process is generated according to equation (5) with $\varepsilon_{t}=\eta_{t} \sigma_{t}$, where $\eta_{t}$ is an i.i.d. $\mathrm{N}(0,1)$ and $\sigma_{t}$ is the scale of variance at t: for an abrupt break

$$
\sigma_{t}= \begin{cases}\sigma_{1} & \text { for } t \leq T_{1}, \\ \sigma_{2} & \text { for } T_{1}<t\end{cases}
$$

and for a series of smooth breaks between $\mathrm{T}_{1}$ and $\mathrm{T}_{2}$,

$$
\sigma_{t}= \begin{cases}\sigma_{1} & \text { for } t \leq T_{1}, \\ \sigma_{1}+\left(\frac{\sigma_{2}-\sigma_{1}}{T_{2}-T_{1}}\right)\left(t-T_{1}\right) & \text { for } T_{1}<t \leq T_{2} \\ \sigma_{2} & \text { for } T_{2}<t\end{cases}
$$

Denoting the break ratio $\left(\sigma_{2} / \sigma_{1}\right)$ as $\delta$, the values $\delta \in\{0.25,0.4,0.6\}$ are considered. Four cases of breaks are examined: CASE I assumes a single big break arising right after $\mathrm{T}_{1}$; CASE II CASE IV witness a sequence of small breaks of same size $\left(\left(\sigma_{2}-\sigma_{1}\right) /\left(T_{2}-T_{1}\right)\right)$ between $T_{1}$ and $T_{2}$. Breaks start at either $\mathrm{T}_{1}=1$ or $\mathrm{T}_{1}=0.1 * \mathrm{~T}+1$, and for CASE II CASE IV they end at $\mathrm{T}_{2}=\mathrm{T}_{1}+0.1 * \mathrm{~T}-1$ (CASE II), $\mathrm{T}_{2}=\mathrm{T}_{1}+0.2 * \mathrm{~T}-1$ (CASE III), or $\mathrm{T}_{2}=\mathrm{T}_{1}+0.3 * \mathrm{~T}-1$ (CASE IV), where $\mathrm{T}$ is either 100 or 500 . Since the initial effect is not a concern, $d=0$ in (5). The simulation result of rejection frequency for the DF-type tests at the $5 \%$ significance level is given in Table 2.

From Table 2 it is clear that early downward breaks cause significant size distortion for the standard DF test regardless of whether the breaks are one-shot or sequential. Actually, sequential small breaks can cause more size distortion than 
an abrupt large break if the breaks begin at $\mathrm{T}_{1}=1$ while the opposite is true if the breaks occur later. On the other hand, variance breaks of all sorts bring about much less serious size problems for the modified DF tests. 
Table 1: Rejection frequency at 5\% level in the presence of GARCH

[a] $\mathrm{T}=100$

\begin{tabular}{c|c|cccc|cccc} 
& & \multicolumn{5}{|c|}{ Initial effect untreated } & \multicolumn{4}{c}{ Initial effect off-loaded } \\
\cline { 3 - 9 }$\left(\phi_{1}, \phi_{2}\right)$ & $\phi_{1}+\phi_{2}$ & DF & DFGLS & DFWS & DFREC & DF & DFGLS & DFWS & DFREC \\
\hline$(0.300,0.650)$ & 0.950 & 0.092 & 0.067 & 0.065 & 0.069 & 0.080 & 0.070 & 0.067 & 0.075 \\
$(0.200,0.750)$ & 0.950 & 0.077 & 0.063 & 0.061 & 0.066 & 0.071 & 0.066 & 0.064 & 0.067 \\
$(0.100,0.850)$ & 0.950 & 0.059 & 0.058 & 0.052 & 0.055 & 0.062 & 0.055 & 0.053 & 0.055 \\
$(0.000,0.950)$ & 0.950 & 0.053 & 0.049 & 0.048 & 0.051 & 0.048 & 0.050 & 0.047 & 0.050 \\
\hline$(0.340,0.650)$ & 0.990 & 0.173 & 0.079 & 0.088 & 0.081 & 0.095 & 0.080 & 0.078 & 0.086 \\
$(0.240,0.750)$ & 0.990 & 0.127 & 0.067 & 0.070 & 0.065 & 0.087 & 0.074 & 0.071 & 0.076 \\
$(0.140,0.850)$ & 0.990 & 0.087 & 0.057 & 0.058 & 0.057 & 0.072 & 0.064 & 0.062 & 0.067 \\
$(0.040,0.950)$ & 0.990 & 0.055 & 0.053 & 0.049 & 0.054 & 0.055 & 0.050 & 0.049 & 0.053 \\
\hline$(0.345,0.650)$ & 0.995 & 0.223 & 0.077 & 0.091 & 0.071 & 0.099 & 0.080 & 0.083 & 0.090 \\
$(0.245,0.750)$ & 0.995 & 0.168 & 0.068 & 0.075 & 0.060 & 0.093 & 0.072 & 0.068 & 0.075 \\
$(0.145,0.850)$ & 0.995 & 0.101 & 0.059 & 0.059 & 0.056 & 0.076 & 0.063 & 0.063 & 0.067 \\
$(0.045,0.950)$ & 0.995 & 0.056 & 0.049 & 0.050 & 0.052 & 0.053 & 0.051 & 0.045 & 0.049 \\
\hline$(0.349,0.650)$ & 0.999 & 0.361 & 0.097 & 0.128 & 0.084 & 0.100 & 0.079 & 0.079 & 0.084 \\
$(0.249,0.750)$ & 0.999 & 0.250 & 0.082 & 0.097 & 0.070 & 0.094 & 0.074 & 0.074 & 0.079 \\
$(0.149,0.850)$ & 0.999 & 0.135 & 0.064 & 0.065 & 0.053 & 0.080 & 0.058 & 0.055 & 0.060 \\
$(0.049,0.950)$ & 0.999 & 0.063 & 0.058 & 0.054 & 0.056 & 0.056 & 0.055 & 0.052 & 0.053
\end{tabular}

[b] $\mathrm{T}=500$

\begin{tabular}{c|c|cccc|cccc} 
& & \multicolumn{5}{|c|}{ Initial effect untreated } & \multicolumn{4}{c}{ Initial effect off-loaded } \\
\cline { 3 - 9 }$\left(\phi_{1}, \phi_{2}\right)$ & $\phi_{1}+\phi_{2}$ & DF & DFGLS & DFWS & DFREC & DF & DFGLS & DFWS & DFREC \\
\hline$(0.300,0.650)$ & 0.950 & 0.077 & 0.065 & 0.072 & 0.075 & 0.075 & 0.063 & 0.070 & 0.074 \\
$(0.200,0.750)$ & 0.950 & 0.071 & 0.059 & 0.068 & 0.068 & 0.069 & 0.059 & 0.066 & 0.067 \\
$(0.100,0.850)$ & 0.950 & 0.055 & 0.054 & 0.053 & 0.054 & 0.056 & 0.052 & 0.051 & 0.052 \\
$(0.000,0.950)$ & 0.950 & 0.048 & 0.045 & 0.049 & 0.047 & 0.050 & 0.050 & 0.050 & 0.049 \\
\hline$(0.340,0.650)$ & 0.990 & 0.142 & 0.073 & 0.093 & 0.088 & 0.101 & 0.080 & 0.094 & 0.101 \\
$(0.240,0.750)$ & 0.990 & 0.123 & 0.065 & 0.081 & 0.081 & 0.092 & 0.079 & 0.086 & 0.088 \\
$(0.140,0.850)$ & 0.990 & 0.090 & 0.065 & 0.075 & 0.074 & 0.084 & 0.069 & 0.075 & 0.077 \\
$(0.040,0.950)$ & 0.990 & 0.054 & 0.053 & 0.053 & 0.052 & 0.055 & 0.056 & 0.056 & 0.056 \\
\hline$(0.345,0.650)$ & 0.995 & 0.188 & 0.077 & 0.102 & 0.098 & 0.104 & 0.082 & 0.093 & 0.098 \\
$(0.245,0.750)$ & 0.995 & 0.174 & 0.077 & 0.104 & 0.093 & 0.104 & 0.078 & 0.091 & 0.097 \\
$(0.145,0.850)$ & 0.995 & 0.133 & 0.069 & 0.080 & 0.073 & 0.090 & 0.069 & 0.076 & 0.078 \\
$(0.045,0.950)$ & 0.995 & 0.060 & 0.053 & 0.054 & 0.051 & 0.065 & 0.058 & 0.056 & 0.057 \\
\hline$(0.349,0.650)$ & 0.999 & 0.358 & 0.087 & 0.146 & 0.109 & 0.108 & 0.082 & 0.099 & 0.102 \\
$(0.249,0.750)$ & 0.999 & 0.336 & 0.082 & 0.130 & 0.094 & 0.105 & 0.084 & 0.098 & 0.102 \\
$(0.149,0.850)$ & 0.999 & 0.239 & 0.075 & 0.101 & 0.075 & 0.096 & 0.068 & 0.080 & 0.081 \\
$(0.049,0.950)$ & 0.999 & 0.089 & 0.055 & 0.061 & 0.057 & 0.069 & 0.053 & 0.057 & 0.057
\end{tabular}




\section{[c] $\mathrm{T}=1000$}

\begin{tabular}{c|c|cccc|cccc} 
& & \multicolumn{5}{|c|}{ Initial effect untreated } & \multicolumn{4}{c}{ Initial effect off-loaded } \\
\cline { 3 - 9 }$\left(\phi_{1}, \phi_{2}\right)$ & $\phi_{1}+\phi_{2}$ & DF & DFGLS & DFWS & DFREC & DF & DFGLS & DFWS & DFREC \\
\hline$(0.300,0.650)$ & 0.950 & 0.076 & 0.062 & 0.074 & 0.083 & 0.077 & 0.070 & 0.074 & 0.082 \\
$(0.200,0.750)$ & 0.950 & 0.064 & 0.063 & 0.067 & 0.073 & 0.068 & 0.062 & 0.065 & 0.073 \\
$(0.100,0.850)$ & 0.950 & 0.057 & 0.054 & 0.055 & 0.060 & 0.058 & 0.054 & 0.057 & 0.064 \\
$(0.000,0.950)$ & 0.950 & 0.049 & 0.055 & 0.050 & 0.053 & 0.054 & 0.054 & 0.051 & 0.055 \\
\hline$(0.340,0.650)$ & 0.990 & 0.127 & 0.077 & 0.094 & 0.100 & 0.104 & 0.084 & 0.099 & 0.109 \\
$(0.240,0.750)$ & 0.990 & 0.115 & 0.074 & 0.089 & 0.094 & 0.096 & 0.083 & 0.089 & 0.101 \\
$(0.140,0.850)$ & 0.990 & 0.081 & 0.065 & 0.073 & 0.079 & 0.080 & 0.067 & 0.076 & 0.081 \\
$(0.040,0.950)$ & 0.990 & 0.058 & 0.054 & 0.056 & 0.061 & 0.062 & 0.053 & 0.057 & 0.060 \\
\hline$(0.345,0.650)$ & 0.995 & 0.160 & 0.083 & 0.108 & 0.109 & 0.103 & 0.088 & 0.098 & 0.110 \\
$(0.245,0.750)$ & 0.995 & 0.153 & 0.080 & 0.102 & 0.105 & 0.107 & 0.084 & 0.096 & 0.107 \\
$(0.145,0.850)$ & 0.995 & 0.120 & 0.070 & 0.087 & 0.092 & 0.094 & 0.074 & 0.085 & 0.093 \\
$(0.045,0.950)$ & 0.995 & 0.066 & 0.057 & 0.059 & 0.066 & 0.062 & 0.059 & 0.058 & 0.064 \\
\hline$(0.349,0.650)$ & 0.999 & 0.302 & 0.083 & 0.130 & 0.115 & 0.115 & 0.084 & 0.101 & 0.116 \\
$(0.249,0.750)$ & 0.999 & 0.304 & 0.092 & 0.131 & 0.113 & 0.108 & 0.083 & 0.097 & 0.109 \\
$(0.149,0.850)$ & 0.999 & 0.243 & 0.079 & 0.109 & 0.097 & 0.106 & 0.081 & 0.090 & 0.099 \\
$(0.049,0.950)$ & 0.999 & 0.106 & 0.060 & 0.068 & 0.068 & 0.081 & 0.063 & 0.064 & 0.068
\end{tabular}

\section{[d] $\mathrm{T}=2000$}

\begin{tabular}{c|c|cccc|cccc} 
& & \multicolumn{5}{|c|}{ Initial effect untreated } & \multicolumn{4}{c}{ Initial effect off-loaded } \\
\cline { 3 - 9 }$\left(\phi_{1}, \phi_{2}\right)$ & $\phi_{1}+\phi_{2}$ & DF & DFGLS & DFWS & DFREC & DF & DFGLS & DFWS & DFREC \\
\hline$(0.300,0.650)$ & 0.950 & 0.065 & 0.058 & 0.064 & 0.068 & 0.069 & 0.057 & 0.065 & 0.070 \\
$(0.200,0.750)$ & 0.950 & 0.060 & 0.056 & 0.057 & 0.057 & 0.058 & 0.058 & 0.058 & 0.060 \\
$(0.100,0.850)$ & 0.950 & 0.055 & 0.056 & 0.057 & 0.057 & 0.056 & 0.050 & 0.052 & 0.053 \\
$(0.000,0.950)$ & 0.950 & 0.046 & 0.051 & 0.049 & 0.049 & 0.050 & 0.050 & 0.051 & 0.050 \\
\hline$(0.340,0.650)$ & 0.990 & 0.108 & 0.078 & 0.091 & 0.095 & 0.094 & 0.074 & 0.092 & 0.098 \\
$(0.240,0.750)$ & 0.990 & 0.102 & 0.073 & 0.089 & 0.091 & 0.091 & 0.076 & 0.087 & 0.093 \\
$(0.140,0.850)$ & 0.990 & 0.083 & 0.064 & 0.073 & 0.077 & 0.081 & 0.068 & 0.074 & 0.080 \\
$(0.040,0.950)$ & 0.990 & 0.054 & 0.050 & 0.052 & 0.053 & 0.054 & 0.053 & 0.054 & 0.053 \\
\hline$(0.345,0.650)$ & 0.995 & 0.128 & 0.076 & 0.094 & 0.097 & 0.105 & 0.083 & 0.094 & 0.103 \\
$(0.245,0.750)$ & 0.995 & 0.128 & 0.080 & 0.098 & 0.101 & 0.103 & 0.080 & 0.093 & 0.099 \\
$(0.145,0.850)$ & 0.995 & 0.106 & 0.075 & 0.087 & 0.089 & 0.083 & 0.070 & 0.080 & 0.085 \\
$(0.045,0.950)$ & 0.995 & 0.065 & 0.057 & 0.057 & 0.059 & 0.059 & 0.058 & 0.059 & 0.059 \\
\hline$(0.349,0.650)$ & 0.999 & 0.229 & 0.080 & 0.112 & 0.101 & 0.106 & 0.082 & 0.099 & 0.110 \\
$(0.249,0.750)$ & 0.999 & 0.231 & 0.079 & 0.111 & 0.101 & 0.107 & 0.088 & 0.103 & 0.112 \\
$(0.149,0.850)$ & 0.999 & 0.214 & 0.082 & 0.103 & 0.095 & 0.109 & 0.087 & 0.098 & 0.104 \\
$(0.049,0.950)$ & 0.999 & 0.104 & 0.061 & 0.071 & 0.067 & 0.086 & 0.065 & 0.069 & 0.069
\end{tabular}


Table 2: Rejection frequency at the $5 \%$ level with variance breaks

[a] Variance breaks starting at $\mathrm{T}_{1}=1$

\begin{tabular}{c|c|cccc|cccc}
\multicolumn{2}{c}{} & \multicolumn{6}{c|}{ T=100 } & \multicolumn{4}{c}{ T=500 } \\
\cline { 3 - 9 } \multicolumn{2}{c}{} & DF & DFGLS & DFWS & DFREC & DF & DFGLS & DFWS & DFREC \\
\hline \multirow{3}{*}{$\delta=0.25$} & CASE I & 0.149 & 0.041 & 0.046 & 0.039 & 0.073 & 0.043 & 0.048 & 0.046 \\
& CASE II & 0.366 & 0.076 & 0.093 & 0.048 & 0.367 & 0.064 & 0.095 & 0.051 \\
& CASE III & 0.379 & 0.086 & 0.107 & 0.064 & 0.373 & 0.080 & 0.113 & 0.064 \\
& CASE IV & 0.323 & 0.089 & 0.108 & 0.066 & 0.315 & 0.083 & 0.113 & 0.064 \\
\hline \multirow{3}{*}{$\delta=0.4$} & CASE I & 0.088 & 0.046 & 0.048 & 0.048 & 0.057 & 0.047 & 0.049 & 0.046 \\
& CASE II & 0.184 & 0.051 & 0.057 & 0.041 & 0.180 & 0.048 & 0.059 & 0.040 \\
& CASE III & 0.215 & 0.055 & 0.063 & 0.042 & 0.209 & 0.055 & 0.065 & 0.045 \\
& CASE IV & 0.208 & 0.061 & 0.066 & 0.047 & 0.203 & 0.059 & 0.068 & 0.046 \\
\hline \multirow{2}{*}{$\delta=0.6$} & CASE I & 0.069 & 0.050 & 0.048 & 0.051 & 0.049 & 0.044 & 0.045 & 0.045 \\
& CASE II & 0.087 & 0.046 & 0.047 & 0.047 & 0.074 & 0.040 & 0.045 & 0.042 \\
& CASE III & 0.107 & 0.045 & 0.049 & 0.047 & 0.094 & 0.041 & 0.046 & 0.041 \\
& CASE IV & 0.117 & 0.046 & 0.050 & 0.046 & 0.102 & 0.040 & 0.048 & 0.040
\end{tabular}

[b] Variance breaks starting at $\mathrm{T}_{1}=0.1 * \mathrm{~T}+1$

\begin{tabular}{c|c|cccc|cccc}
\multicolumn{2}{c}{} & \multicolumn{6}{|c|}{ T=100 } & \multicolumn{4}{c}{ T=500 } \\
\cline { 3 - 9 } \multicolumn{2}{c|}{} & DF & DFGLS & DFWS & DFREC & DF & DFGLS & DFWS & DFREC \\
\hline \multirow{3}{*}{$\delta=0.25$} & CASE I & 0.400 & 0.079 & 0.102 & 0.055 & 0.406 & 0.080 & 0.120 & 0.061 \\
& CASE II & 0.398 & 0.095 & 0.116 & 0.066 & 0.399 & 0.084 & 0.124 & 0.073 \\
& CASE III & 0.336 & 0.095 & 0.114 & 0.070 & 0.331 & 0.083 & 0.117 & 0.071 \\
& CASE IV & 0.267 & 0.083 & 0.099 & 0.069 & 0.260 & 0.077 & 0.108 & 0.068 \\
\hline \multirow{3}{*}{$\delta=0.4$} & CASE I & 0.228 & 0.057 & 0.066 & 0.045 & 0.225 & 0.053 & 0.067 & 0.045 \\
& CASE II & 0.239 & 0.066 & 0.072 & 0.048 & 0.230 & 0.057 & 0.072 & 0.046 \\
& CASE III & 0.223 & 0.066 & 0.073 & 0.051 & 0.213 & 0.060 & 0.073 & 0.045 \\
& CASE IV & 0.199 & 0.069 & 0.075 & 0.053 & 0.190 & 0.058 & 0.072 & 0.047 \\
\hline \multirow{3}{*}{$\delta=0.6$} & CASE I & 0.122 & 0.050 & 0.049 & 0.043 & 0.116 & 0.051 & 0.055 & 0.044 \\
& CASE II & 0.126 & 0.050 & 0.050 & 0.043 & 0.120 & 0.050 & 0.058 & 0.044 \\
& CASE III & 0.128 & 0.050 & 0.052 & 0.043 & 0.122 & 0.053 & 0.058 & 0.045 \\
& CASE IV & 0.131 & 0.051 & 0.054 & 0.044 & 0.120 & 0.053 & 0.059 & 0.044
\end{tabular}

Note. CASE I: a one-shot variance break at $\mathrm{T}_{1}$; CASE II: sequential breaks starting at $\mathrm{T}_{1}$ and ending at $\mathrm{T}_{2}=\mathrm{T}_{1}+0.1^{*} \mathrm{~T}-1$; CASE III: sequential breaks starting at $\mathrm{T}_{1}$ and ending at $\mathrm{T}_{2}=\mathrm{T}_{1}+0.2 * \mathrm{~T}-1$; CASE IV: sequential breaks starting at $\mathrm{T}_{1}$ and ending at $\mathrm{T}_{2}=\mathrm{T}_{1}+0.3 * \mathrm{~T}-1$. 